35 OBSERVATIONAL STUDY TO DETERMINE THE PREVALENCE OF TRANSTHYRETIN AMYLOIDOSIS IN AN UNDIFFERENTIATED HEART FAILURE WITH PRESERVED EJECTION FRACTION POPULATION

${ }^{1} \mathrm{G}$ Giblin, ${ }^{1} \mathrm{~A}$ Gray, ${ }^{1} \mathrm{~L}$ Murphy, ${ }^{1} \mathrm{E}$ Kavanagh, ${ }^{1} \mathrm{C}$ Tracey, ${ }^{1} \mathrm{C}$ Howley, ${ }^{2} \mathrm{~N}$ Murphy. ${ }^{1} \mathrm{O} \mathrm{O}^{\prime} \mathrm{Neill}$; ${ }^{1} M O^{\prime}$ Connell; ${ }^{1} M$ Coyne; ${ }^{1} E$ Joyce; ${ }^{1} N$ Mahon; ${ }^{1}$ Mater Misericordiae University Hospital, Dublin, Ireland; ${ }^{2}$ Our Lady of Lourdes, Drogheda, Ireland

\subsection{6/heartjn|-2020-ICS.35}

Introduction Heart failure with preserved ejection fraction (HFpEF) is a common but heterogenous clinical syndrome. Transthyretin amyloidosis cardiomyopathy (ATTR-CM) can now be diagnosed non-invasively with high sensitivity incorporating Technitium-99m, 3,3-diphosphono-1,2-propanodicarboxylic acid (Tc-DPD) scintigraphy. ATTR-CM has been found as the underlying aetiology of heart failure in $13 \%$ in $\mathrm{HFpEF}$ syndrome with left ventricular hypertrophy (LVH). Timely diagnosis of ATTR-CM has become even more relevant given the recent availability of targeted treatments. However, the prevalence and spectrum of ATTR-CM has not previously been studied in all-comer HFpEF cohort.

Aims To determine the prevalence and spectrum of ATTR-CM in an undifferentiated multicentre HFpEF population using the noninvasive diagnostic pathway incorporating Tc-DPD scintigraphy.

Methods Consecutive patients $\geq 60$ years attending the heart failure services with a diagnosis of HFpEF and New York Heart Association class II-IV symptoms across 4 sites were prospectively enrolled. Severe valvular pathology or a prior history of symptomatic myeloma, AL amyloidosis or monoclonal gammopathy of unknown significance (MGUS) were excluded. Transthoracic 2D strain echocardiography, 12 lead electrocardiogram (ECG), biomarker analysis and Tc-DPD scintigraphy were performed. Tc-DPD findings were graded visually according to the Perugini classification (grades 0-3). Those patients with Perugini grade $2-3$ on Tc-DPD (moderatesevere uptake; highly suggestive of ATTR-CM) underwent additional laboratory and haematological assessment and transthyretin (TTR) gene sequencing.

Results To date, 45 patients $(49 \%$ female, mean age $77.6 \pm$ 7.9 years) have been prospectively enrolled. A history of atrial fibrillation and hypertension were present in $60 \%$ and $80 \%$ respectively. In keeping with ATTR-CM, no participant had a low voltage pattern on ECG. Three patients (6.7\%) had Perugini grade 3 uptake on Tc-DPD and 6 patients (13.3\%) had grade 1 uptake (mild uptake, inferior to bone; diagnostic significance undetermined). No patients had grade 2 uptake. All those with grade 3 uptake had AL amyloid excluded on further haematological testing and no mutation was detected in the TTR gene confirming wild type ATTR-CM. Significant differences across Tc-DPD uptake grades were seen according to increasing age and LV wall thickness (figure 1).

Conclusion ATTR-CM, characterised by high grade uptake (Perugini 3) on Tc-DPD scintigraphy and negative haematological markers, was present in $6.7 \%$ of this undifferentiated HFpEF cohort and was associated with older age, and increased left ventricular wall thickness. Low grade uptake (Perugini grade 1) was present in a further $13.3 \%$. While the diagnostic significance of this phenotype is undetermined, this may represent early ATTR-CM, suggesting that Tc-DPD scintigraphy may be able to identify a spectrum of disease according to uptake that correlates with wall thickness. Further follow up in this study will explore this potential.

\section{IDENTIFICATION AND MANAGEMENT OF PATIENTS WITH ELEVATED BMI IN ST. JAMES'S HOSPITAL}

${ }^{1} \mathrm{C}$ Balfe, ${ }^{2} \mathrm{R}$ Corcoran, ${ }^{2} \mathrm{~A}$ GilmartinR Nadarajan. ${ }^{1}$ Tallaght University Hospital, Dublin, Ireland; 'St James's Hospital, Dublin, Ireland

\subsection{6/heartjnl-2020-ICS.36}

The rapid rise in rates of obesity in recent decades represents a major challenge in healthcare provision. In Ireland in 2011, $26 \%$ of men and 21\% of women were obese. Analysis from the United Kingdom has suggested that by 2050 , over half of the adult population may be obese. BMI is widely used to classify individuals as normal weight, underweight, overweight and obese. Severe obesity is defined as a BMI $>40 \mathrm{~kg} / \mathrm{m}^{2}$ (or $\geq 35 \mathrm{~kg} / \mathrm{m}^{2}$ in the presence of comorbidities).

We analysed a group of patients admitted to the acute medical assessment unit in St. James's Hospital on unselected general medical take in July and August 2019. We measured the height, weight and body mass index of each patient and reviewed the medical notes to identify if abnormal BMIs were being identified and discussed with patients.

We identified the proportion of patients with an elevated BMI who were informed of their condition, and who had a management plan discussed by their primary medical team. We additionally determined if patients with an elevated BMI were being referred to a dietician service. 74 patients $(39$ men

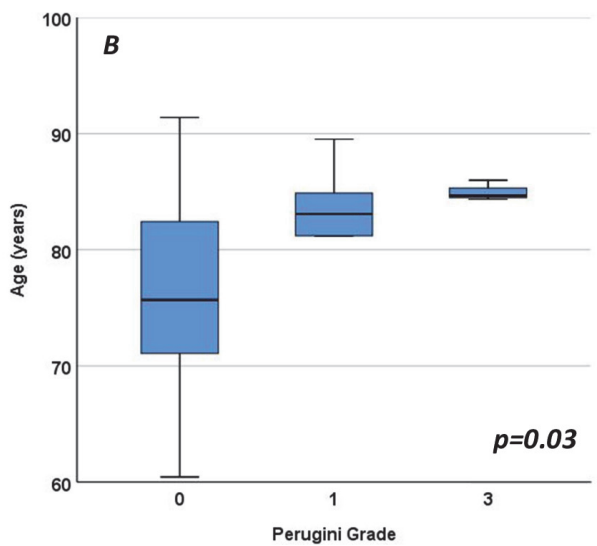

Abstract 35 Figure 1 Distribution of (A) LV wall thickness and (B) age by DPD Perugini Grade 
and 35 women) were included in the final analysis. Given the setting of unselected intake, there was significant heterogeneity in the weights of the cohort assessed, with inclusion of patients with low and very elevated BMIs. The BMI spread from the cohort is displayed in figure 1.

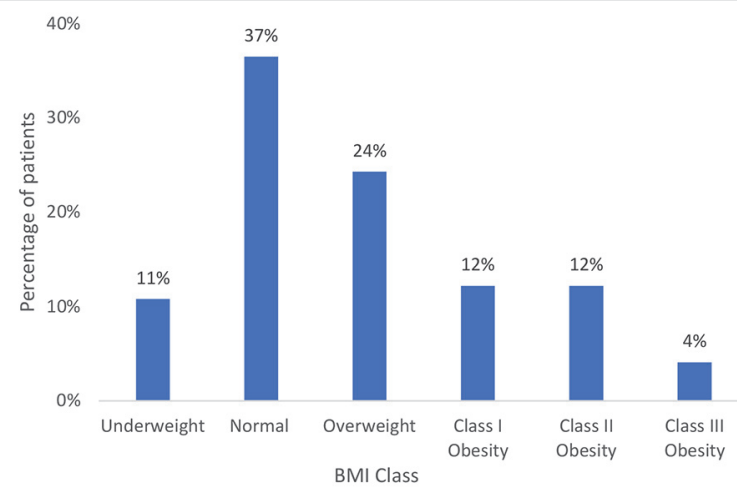

Abstract 36 Figure 1 BMI spread from the cohort of 74 patients

We considered a patient's weight to have been addressed by the primary medical team if an overweight or obese status was discussed with the patient and/or documented in the admission note. 10 out of 39 patients who were overweight or obese had this documented in their admission note (26\%). No patient had their BMI measured independent of a nutritionist's input. Referral to dietetics was made in $75 \%$ of underweight patients, $33 \%$ of overweight patients, $11 \%$ of patients with class I obesity, $66 \%$ of those with class II obesity and $66 \%$ of those with class III obesity. 4\% of patients had weight loss proposed in their management plan. Hypertension was a comorbidity in $50 \%$ of patients, with $34 \%$ of patients having a diagnosis of dyslipidaemia. Musculoskeletal disease was present in $26 \%$, most commonly in the form of gout. Documentation of a patient's weight is standard practice during a modern day hospital admission, mainly due to the necessity for weight-based medication dosing and accurate assessment of renal function. However, as it stands it is not the routine to calculate the body mass index during these same admissions. Nonetheless, calculation of the BMI represents the prerequisite to identifying and advocating for patients who are overweight and obese. We argue that it would be optimal for all admitted patients to have their height and weight measured during their admission, with subsequent calculation of the BMI. With an electronic patient record (due to be initiated in all public hospitals in Ireland in the coming years), the BMI could be published on every patients home page. We feel that this would act as an impetus to discussion and management of an often under acknowledged comorbidity.

\section{ASSOCIATION BETWEEN PRE-OPERATIVE CARDIOVASCULAR RISK FACTORS AND CORONARY ARTERY CALCIUM SCORES IN PATIENTS UNDERGOING BARIATRIC SURGERY}

IJ Schoenberg, ${ }^{1,2} \mathrm{C}$ O'Boyle, ${ }^{1,2} \mathrm{CJ}$ O'Sullivan. ${ }^{1}$ University College Cork, Cork, Ireland; ${ }^{2}$ Bon Secours Hospital, Cork, Ireland

10.1136/heartjnl-2020-ICS.37
Background Obesity is associated with many factors that predispose to cardiovascular disease, including hypertension, dyslipidaemia, and diabetes. Bariatric surgery has been shown to decrease cardiovascular disease risk. Coronary artery calcium scoring (CACS) is a non-invasive imaging technique that quantifies intra-arterial calcium resulting from atherosclerosis. Elevated CACS has been shown to be correlated with cardiovascular disease progression and morbidity. Currently it is not well established whether morbidly obese patients carry a coronary artery calcium burden or which factors are associated with a higher CACS in a bariatric surgical population pre-operatively.

Methods Patients with no prior cardiovascular disease undergoing bariatric surgery were evaluated preoperatively for CACS. Age, sex, smoking status, diabetes status, blood pressure, serum lipids, pre- and post- operative weight and height were also recorded. A ten-year risk of fatal cardiovascular disease was calculated using the European Society of Cardiology's SCORE tool. Patients were grouped into CACS $=0$ and CACS $>0$. Statistical analysis was performed utilizing the Student's T-test for continuous variables and Chi Square test for categorical variables. P-values less than 0.05 were considered statistically significant.

Results Between June 2008 and June 2019, 154 patients were evaluated. The mean (sd) age was 44(11) years. Thirty-six (23\%) were male. Forty-eight (31\%) patients had subclinical coronary artery calcium scores. Risk factors that were associated with CACS $>0$ were older age (mean 53(6) vs $40(10)$ years, $\mathrm{p}<0.001)$, male sex $(37 \%$ vs $17 \%, \mathrm{p}=0.005)$, diabetes $(27 \%$ vs $12 \%, p=0.023)$, systolic blood pressure (141(17) $\mathrm{mmHg}$ vs $132(17) \mathrm{mmHg}, \mathrm{p}=0.004)$, and hyperlipidaemia requiring treatment $(42 \%$ vs $7 \%, \mathrm{p}<0.001)$. Higher cardiovascular risk scores were associated with CACS $>0$ (mean 1.2 (1.28) vs $0.29(0.73), \mathrm{p}<0.001)$ The absence of CAC pre-operatively was associated with greater excess weight loss at 12 month follow up $(79.71(28) \%$ vs $68.88(22) \%, \mathrm{p}=0.038)$.

Conclusions Our study demonstrates that coronary artery calcium is associated with adverse cardiovascular risk factors in

Abstract 37 Table 1 Clinical and laboratory parameters in pre-operative bariatric surgery patients

\begin{tabular}{|c|c|c|c|c|}
\hline & $\begin{array}{l}\text { All } \\
\text { Participants }\end{array}$ & CAC Score $=0$ & CAC Score $>0$ & P Value \\
\hline Patients, n (\%) & 154 & $106(69)$ & $48(31)$ & \\
\hline Age, mean (SD) & 44.25 (11.44) & $40.11(10.20)$ & $53.29(8.46)$ & $<0.001 *(1$ \\
\hline Sex, male n (\%) & $36(23)$ & $18(17)$ & $18(37)$ & $0.005^{*(1)}$ \\
\hline Smoker, n (\%) & $17(11)$ & $14(13)$ & $3(6)$ & 0.202 \\
\hline Diabetes, n (\%) & $26(17)$ & $13(12)$ & $13(27)$ & $0.023^{*(1)}$ \\
\hline $\begin{array}{l}\text { \%XS Weight loss, } \\
\text { mean (SD) }\end{array}$ & $76.13(27)$ & $79.71(28)$ & $68.88(22)$ & $0.038^{*(2)}$ \\
\hline $\begin{array}{l}\text { BP on medication, } n \\
(\%)\end{array}$ & $57(38)$ & $24(23)$ & $33(70)$ & $<0.001 *(2$ \\
\hline $\begin{array}{l}\text { Systolic BP mmHg, } \\
\text { mean (SD) }\end{array}$ & $\begin{array}{l}134.71 \\
(17.65)\end{array}$ & $132.0(17.35)$ & $140.67(17.00)$ & $0.004^{*(2)}$ \\
\hline $\begin{array}{l}\text { Lipid lowering } \\
\text { medication, n (\%) }\end{array}$ & $24(19)$ & $6(7)$ & $18(42)$ & $<0.001 *(1$ \\
\hline ESC SCORE & & & & \\
\hline Mean (SD) & $0.57(1.02)$ & $0.29(0.733)$ & $1.2(1.28)$ & $<0.001 *(2$ \\
\hline SCORE $=0, n(\%)$ & $99(66)$ & $84(81)$ & $15(33)$ & $<0.001^{*(1}$ \\
\hline SCORE $=1-6, n(\%)$ & $51(33)$ & $20(19)$ & $31(66)$ & \\
\hline
\end{tabular}

